\title{
BMJ Open Quality Improving the care of patients with cystic fibrosis (CF)
}

\author{
Ahsan Aftab Khan, ${ }^{1}$ Edward F Nash, ${ }^{2}$ Joanna Whitehouse, ${ }^{2}$ Rifat Rashid ${ }^{2}$
}

To cite: Khan AA, Nash EF, Whitehouse J, et al. Improving the care of patients with cystic fibrosis (CF). BMJ Open Quality 2017;6:e000020. doi:10.1136/ bmjoq-2017-000020

- Additional material is published online only. To view please visit the journal online (http://dx.doi.org/10.1136/ bmjoq-2017-000020).

Received 6 February 2017 Revised 4 July 2017 Accepted 29 July 2017

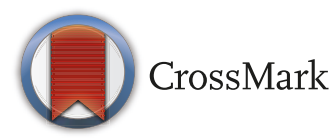

${ }^{1}$ Cardiology Department, Sandwell and West Birmingham Hospitals NHS Trust, Birmingham, UK

${ }^{2}$ Respiratory Medicine, Heart of England NHS Foundation Trust, Birmingham, UK

Correspondence to Dr Ahsan Aftab Khan, Queen Elizabeth Hospital Birmingham, University Hospitals Birmingham NHS Foundation Trust, Mindelsohn Way, Birmingham, West Midlands, UK, B15 2TH; ahsan.khan@hotmail.co.uk

\section{ABSTRACT}

Background The West Midlands Adult Cystic Fibrosis (CF) Centre based at Birmingham Heartlands Hospital provides care for adults with CF in the West Midlands. People with CF are prone to pulmonary exacerbations, which often require inpatient admission for intravenous antibiotics. We observed that the admission process was efficient during working hours (9:00-17:00, Monday-Friday) when the CF team are routinely available, but out-of-working hours, there were delays in these patients being clerked and receiving their first antibiotic dose. We were concerned that this was resulting in quality and potential safety issues by causing delays in starting treatment and prolonging hospital inpatient stays. We therefore undertook a quality improvement project (QIP) aimed at addressing these issues. An initial survey showed median time to clerk of 5 hours, with $60 \%$ of patients missing their first dose of antibiotics and mean length of stay of 16 days.

Methods We applied the Plan-Do-Study-Act (PDSA) cycle approach, with the first PDSA cycle involving raising awareness of the issue through education to doctors, nurses and patients.

Results This led to a reduction of median time to clerk from 5 to 2 hours with $23 \%$ of patients missing their first antibiotic dose and mean length of stay reducing to 14 days. The second cycle involved introducing an admissions checklist and displaying education posters around the hospital, resulting in median time to clerk remaining at 2 hours but only $20 \%$ of patients missing their first antibiotic dose and the mean length of stay remaining at 14 days. Conclusion This QIP has improved the out-of-hours admissions process for adults with $\mathrm{CF}$ in our centre. We plan to review the longer term effects of the project including sustainability, effects on clinical outcomes and patient satisfaction.

\section{PROBLEM}

People with cystic fibrosis $(\mathrm{CF})$ suffer frequent pulmonary exacerbations, requiring treatment with oral or intravenous antibiotics. When intravenous antibiotics are necessary, patient may be admitted to a specialist CF centre for intensive multidisciplinary care. The West Midlands Adult Cystic Fibrosis Centre (WMACFC) based at Birmingham Heartlands Hospital (BHH) cares for adults with CF living in the West Midlands. $\mathrm{BHH}$ is a large teaching hospital at the heart of a bustling, dynamic community in centraleastern Birmingham, England. WMACFC comprises 20 single en-suite rooms, an adjacent outpatient department, a gymnasium and team offices. CF adults requiring intravenous antibiotics are triaged either in outpatients, during home visits by $\mathrm{CF}$ nurses, or via an emergency helpline, and their admission is arranged. On arrival to the CF centre, they should be clerked promptly by a doctor so that they can receive clinical review and be started on intravenous antibiotics without delay. During working hours (9:00-17:00, Monday-Friday), the CF medical team admit these patients in a timely fashion. However, when patients arrive out of hours, they are usually clerked by an on-call doctor who will not usually have received specialist training in CF.

We were concerned that patients with $\mathrm{CF}$ arriving out of hours were frequently not clerked for many hours leading to delays in their treatment. This was felt to be a quality and potential safety issue, since we observed unacceptable delays between potentially unwell patients arriving on the ward and receiving clinical review. Additional potential concerns included delays in receiving treatment, prolonged hospital admission and delayed discharge.

We therefore undertook a quality improvement project (QIP) aiming to explore these issues and improve the admission process for patients with CF. Our aim was that $100 \%$ of patients with CF arriving out of hours (17:009:00, Monday-Friday and weekends) are clerked within 4 hours and prescribed appropriate treatment.

\section{BACKGROUND}

$\mathrm{CF}$ is an autosomal recessive multisystem disorder with an incidence of approximately 1:1500 in North America and most European countries. ${ }^{1}$ It affects more than 10800 people in UK with 2.5 million people being carriers of a CF mutation (about 1 in 25). ${ }^{2}$ It is a life-limiting disease, and despite overall improvements in survival, patients with CF experience frequent pulmonary exacerbations and usually succumb to respiratory failure. ${ }^{3} \mathrm{CF}$ is caused by reduced function of the $\mathrm{CF}$ transmembrane conductance regulator (CFTR), ${ }^{4}$ an ion channel that regulates 
sodium and chloride across epithelial cell membranes. CFTR dysfunction causes dehydrated, sticky secretions, with consequent impairment of mucociliary clearance, ${ }^{5}$ chronic respiratory infection, inflammation and progressive respiratory failure. ${ }^{67}$

Current CF treatments predominantly target respiratory infections, inflammation, mucociliary clearance and nutritional status. ${ }^{1}$ A combination of chest physiotherapy and medication can help control lung infections and prevent the build-up of mucus that causes the damage. ${ }^{2}$ CFTR modulators, targeting the underlying CFTR defect, are an exciting novel class of therapies that are becoming more widely available. A combination of these treatments as part of multidisciplinary care has resulted in increased life expectancy, delayed lung function decline and improved quality of life for patients with $\mathrm{CF}^{8}{ }^{8}$ Optimal treatment of pulmonary exacerbations requires recognition of unwell patients with $\mathrm{CF}$ by the clinicians, proactive admission to a specialised CF unit and prompt initiation of treatment, the mainstay of which is intravenous antibiotics.

\section{BASELINE MEASUREMENT}

Data collection was carried at the WMACFC using a proforma that included: date of admission, time of admission, date of clerking, time of clerking and prescribing of antibiotics, date and time of antibiotic administration and length of stay. This information was obtained from patients' notes and electronic prescribing software. Information was obtained on whether antibiotics were specified in a clinical letter or clerking plan by the CF team to aid the on-call clerking doctor. Baseline data was collected over a period of 2 weeks and included 20 patients.

The data showed an average time delay in clerking of 5 hours with 6 out of 20 patients (30\%) clerked more than 8 hours after presenting to hospital. The appropriate antibiotics to prescribe were documented by the CF team for 14 out of 20 patients $(70 \%)$. Twelve out of 20 patients $(60 \%)$ missed their first dose of antibiotic. The reasons for missing first dose of antibiotics were: delay in gaining intravenous access (one patient), delay in clerking (six patients) and antibiotics not documented by CF team to prescribe (five patients). The mean length of stay for these patients was 16 days.

\section{DESIGN}

The baseline data provided a clear impetus to improve the overall clerking process for these patients. We realised that the main issue was lack of education among staff members about the importance of promptly clerking these patients. Initial feedback through targeted discussions with key staff showed that staff did not realise how unwell these 'planned' admissions could be. It was a feeling among staff especially junior doctors that since these patients were 'planned' admissions, they were more likely to be clinically stable and therefore did not require to be seen urgently. Education was targeted at junior doctors, nursing staff and clerical staff. Other potential solutions were recognised, such as clear documentation of management plans by the CF team for junior doctors with little experience of patients with CF, reminders for patients with CF to arrive to hospital promptly and fostering team spirit by appropriately utilising each team member's skill set, such as nursing staff performing intravenous cannulation to administer antibiotics.

The primary author led the project, supported by the coauthors. A process map outlining the patient journey was developed (see online supplementary figure 1). Intervention at each step of the pathway was applied using a driver diagram (see online supplementary figure 2). When patients were seen in clinic and it was decided that they required admission for intravenous antibiotics, the clinician responsible was asked to advise the patient to arrive on the unit promptly when he/she was informed that a bed was available. Also, the clinician was asked to clearly document the management plan including the name of antibiotics, dosage, route and frequency in the patient's notes. Similarly, patients who were admitted via home visits by the CF specialist nurses or through the emergency helpline were discussed with the CF consultant, and a management plan was put in place to assist the on-call team.

Subsequently, the CF team was informed of the prospective admission so they could identify suitable discharges and free up beds earlier in the day. The patient was called by the specialist CF nurse or clerical staff to advise them to attend the unit as soon as possible. A target for this call was set to midday. A text reminder service was proposed to remind patients about their planned admission date.

Once the patient arrived to the unit, a nurse looking after the patient was required to admit the patient in a timely manner, perform observations to calculate modified early warning score and gain intravenous access. If it was out of hours (17:00-9:00, Monday-Friday and weekends), the ward nurse was required to inform the on-call team to advise them to clerk the patient. It was made clear that the on-call junior doctor was expected to clerk the patient within 4 hours of admission, prescribe the specified antibiotics and inform the nurse, so that antibiotics could be administered promptly.

The first intervention was to educate junior doctors, nurses, ward clerk and CF team (consultants, registrars and specialist nurses) through face-to-face meetings, oral presentation and emails. Junior doctors on the general internal medicine (GIM) on-call rota were sent an email followed by a talk given during their weekly teaching on the importance of clerking patients with CF promptly. The email and the talk covered several topics, such as how unwell these patients could potentially be; the importance of reviewing them soon after their arrival to initiate appropriate treatment; and administering intravenous antibiotics early to prevent morbidity, reduce length of hospital stay and enable other patients to be admitted for treatment sooner. This also allowed us to obtain feedback from key staff members on the admission process and the problems faced in clerking these patients. 
The second intervention was to create an admissions checklist (see online supplementary figure 3). This provided an aide-memoire for nursing and clerical staff to follow steps in admitting the patient to the unit and a place for time stamps once each step was completed. It was the responsibility of the nursing staff to complete the checklist. The checklist also provided an important utility for future audits to subsequently help improve the standards of care. Lastly, we created wall posters to serve as reminders for all stakeholders on the importance of promptly clerking patients with CF being admitted directly to the unit. Two examples of these posters are attached as supplementary files (online supplementary figureS 4 and 5).

We also recognised several limitations that may affect the long-term sustainability of this project. These included the changeover of staff especially junior doctors every 4-6 months, low number of nurses experienced in working on CF unit, which meant agency staff covering gaps in the rota, and no induction for junior doctors and nursing staff at the start of their rotations on the importance of clerking CF admissions promptly. The long-term sustainability was discussed with senior members of the $\mathrm{CF}$ team, and it was agreed to appoint a CF specialist nurse as 'admissions champion' for an ongoing source of support for the QIP.

\section{STRATEGY}

Our SMART (specific, measureable, agreed upon, realistic and time-based) aim was to improve clerking of all $\mathrm{CF}$ admissions to the unit within 4 hours and reduce incidence of missing first dose of antibiotic within 4 months. The 4-hour target was selected as an arbitrary target. The missing first dose of antibiotic referred to the dose of antibiotic that would have been administered to the patient if they were clerked and had intravenous access available before nearest specified antibiotic administration time. Data were collected with the same proforma used for baseline measurements. We carried out two Plan-DoStudy-Act (PDSA) cycles.

PDSA cycle 1: our intervention was to provide education to various stakeholders. We did this by educating nursing staff on a one-to-one basis and as a group during their handover meetings. Education to junior doctors was through one of the CF consultants sending an email to all junior doctors on the GIM on-call rota to explain the importance of clerking patients with CF promptly. This was followed by a short PowerPoint presentation to junior doctors during one of their weekly teaching sessions. A presentation was also delivered to the CF department, which included consultants, registrars, junior doctors, CF specialist nurses, pharmacist and other support staff. The presentation emphasised the importance of accurately documenting suggested intravenous antibiotics for all admissions in patients' notes, to carry out timely discharges on the unit and provide reminders to patients with CF awaiting admission of the importance of prompt attendance. Data were collected over 2 weeks following the intervention and included 13 patients. The average time of clerking was reduced to 2 hours with only $23 \%$ of patients missing their first dose of antibiotics. Feedback obtained from this intervention was largely on methods to ensure sustainability of this progress.

PDSA cycle 2: in order to build and strengthen our progress, an admission checklist was created to accurately record data and serve as an aide-memoire for nursing and clerical staff. This was followed by producing brief A3-sized and A4-sized posters underlining the education aspect and serving as a reminder for patients, doctors and nursing staff. We put these posters in the CF outpatient department, inpatient unit, medication room and the on-call doctors' office on the acute medical unit. Data were collected over 2 weeks following the intervention and included 15 patients. The average time taken to clerk patients with CF remained at 2 hours, and the incidence of first dose of antibiotic missed from $23 \%$ to $20 \%$. Feedback from this cycle was to maintain this progress and implement a CF 'admissions champion' to continue the long-term sustainability of the project. Going forward, one of the areas to explore is to include the educational intervention in the induction for new staff members relevant to the $\mathrm{CF}$ unit.

\section{RESULTS}

Our process measures were: (1) average time taken to clerk CF admissions and (2) number of patients missing their first dose of antibiotic(s). Our outcome measure was average length of hospital stay. The overall results have been extremely positive. The interventions carried out in PDSA cycle 1 led to a reduction in average time taken to clerk patients from baseline measurement of 5 hours to 2 hours. The antibiotics were documented for $100 \%$ of patients by the CF team compared with $70 \%$ in baseline measurement. Twenty-three per cent of patients missed their first dose of antibiotic, which was a significant improvement from $60 \%$. The average length of stay was reduced from 16 to 14 days.

Following PDSA cycle 2, the average time taken to clerk remained at 2 hours. The antibiotics were again documented for $100 \%$ of patients. However, the incidence of missing first dose of antibiotics in patients was further reduced to $20 \%$. The average length of stay remained at 14 days. See supplementary file.

\section{LESSONS AND LIMITATIONS}

We found it extremely helpful to develop a process map (online supplementary figure 1) to cover a typical journey of a patients with CF from being seen in the clinic to admission to the ward. This allowed us to prepare a driver diagram (online supplementary figure 2) and break each process down to single steps to target interventions at multiple levels and therefore achieve the best possible outcome. The PDSA cycle approach allowed us to test an intervention in a short period of time and assess its respective suitability and efficacy. 
We identified all stakeholders in the process of clerking patients with $\mathrm{CF}$, which included the patients, nursing staff, junior doctors on GIM on-call rota and CF department. This allowed us to involve all of them in our interventions. The interventions were mainly education based, highlighting this quality and potential safety issue. By involving all stakeholders in our project, we were able to listen to their feedback, increase their engagement with the project and subsequently increase the chances of its success.

Our project did have several limitations. The main limitation was small sample sizes in each PDSA cycles. Another important limitation was the variation in experience of different junior doctors in managing patients with CF. For example, junior doctors with previous experience of looking after patients with CF were more likely to attend to these patients in a timely manner. This could have perhaps contributed to the improvement seen in our data. There is also the potential for the results to be affected by positive confounding bias, for example, due to the change in doctors on GIM on-call rota every 4 months. Other issues include patients sometimes refusing to allow intravenous access to be obtained, staff not trained to use the long-term intravenous access and a particular antibiotic being out of stock or not available on the unit. Lastly, the small number of data points in our PDSA cycles prevented us from performing meaningful statistical analysis to determine significance.

As each of our interventions showed improvement, we did not feel the need to modify our interventions but rather consolidate on them, for example by incorporating the educational intervention in the induction programmes for prospective nurses and junior doctors to ensure long-term sustainability. This would be overseen by a CF specialist nurse who will act as an 'admissions champion' for this process. We also identified that the admission checklist was not available on the ward for a few weeks as the ward clerk responsible to print it and place it in patients' notes was on leave. This meant that admission checklist was not completed for some patients. To avoid this in the future, senior nurses and other ward clerks who provide cover in the absence of the main clerk for the unit were given induction on the use of the admission checklist.

In order to maintain our progress on a long-term basis, a suggestion has been made to have a designated on-call doctor for the CF unit during out of hours but that would need negotiation and funding at a Trust level. Data collection was ceased due to the primary author changing rotation to a different hospital, but we hope to continue data collection by the admissions champion with support from junior doctors in the current academic year.

\section{CONCLUSION}

The project's aim was to improve the clerking process for patients with CF who are admitted directly to the CF unit to receive intravenous antibiotics for a pulmonary exacerbation. We identified a quality and potential patient safety issue, since a proportion of patiets were not being clerked for several hours following admission, leading to delays in receiving their antibiotics on time. One of the main reasons behind this was the lack of awareness among staff looking after these patients and thus our interventions in this QIP aimed at resolving this. A sustainable approach was applied to address this issue.

This project has demonstrated improvement in the standards of care for patients with CF through multiple, cost-effective, conducive and efficient interventions. The results have been extremely encouraging, and we achieved our SMART aim within 1 PDSA cycle. While recognising the significant progress made, we understand that continued work is required to ensure standards are met and improved further. To sustain the continued improvement and avoid a drift back to previous practice, a role of an admissions champion has been established to continue the work and engage all stakeholders in improving the outcomes further. The significant results gained from the project has provided a platform for other departments within the same hospital to adopt this practice to enable improvement in standards of care for clerking planned admissions, especially in departments such as haematology, oncology, nephrology, cardiology and infectious diseases.

Through this project, we have shown how an organised approach to a problem can result in multiple solutions targeted at several steps of the pathway and achieve positive outcomes. We believe that our approach can be easily replicated by other centres experiencing similar problems.

Acknowledgements We would like to thank Dr Adel Mansur (respiratory consultant) and Dr Claire Baker (cystic fibrosis clinical fellow).

Contributors This quality improvement project report was produced by the primary author and coauthors with no contribution from any other individual.

Competing interests None declared.

Provenance and peer review Not commissioned; externally peer reviewed.

Open Access This is an Open Access article distributed in accordance with the Creative Commons Attribution Non Commercial (CC BY-NC 4.0) license, which permits others to distribute, remix, adapt, build upon this work non-commercially, and license their derivative works on different terms, provided the original work is properly cited and the use is non-commercial. See: http://creativecommons.org/ licenses/by-nc/4.0/

(C) Published by the BMJ Publishing Group Limited. For permission to use (where not already granted under a licence) please go to http://www.bmj.com/company/ products-services/rights-and-licensing/

\section{REFERENCES}

1. Zemanick ET, Harris JK, Conway S, et al. Measuring and improving respiratory outcomes in cystic fibrosis lung disease: opportunities and challenges to therapy. $J$ Cyst Fibros 2010;9:1-16.

2. Cystic Fibrosis Trust. What is CF? https://www.cysticfibrosis.org.uk/ what-is-cystic-fibrosis

3. Gibson RL, Burns JL, Ramsey BW. Pathophysiology and management of pulmonary infections in cystic fibrosis. Am J Respir Crit Care Med 2003;168:918-51.

4. Davis PB, Drumm M, Konstan MW. Cystic fibrosis. Am J Respir Crit Care Med 1996;154:1229-56. 
5. Puchelle E, Bajolet $\mathrm{O}$, Abély M. Airway mucus in cystic fibrosis. Paediatr Respir Rev 2002;3:115-9.

6. Flume PA, Mogayzel PJ, Robinson KA, et al. Cystic fibrosis pulmonary guidelines: pulmonary complications: hemoptysis and pneumothorax. Am J Respir Crit Care Med 2010;182:298-306.
7. Mitchell RS, Kumar V, Robbins SL, et al. Robbins basic pathology. Saunders/Elsevier 2007.

8. Landon C, Rosenfeld RG. Short stature and pubertal delay in cystic fibrosis. Pediatrician 1987;14:253-60. 